\title{
Unusual Phase Behavior in Mixtures of Poly(ethylene oxide) and Ethyl Alcohol
}

\author{
DEREK L. HO, ${ }^{1,2}$ BOUALEM HAMMOUDA, ${ }^{1}$ STEVEN R. KLINE, ${ }^{1}$ WEI-REN CHEN ${ }^{1,3}$ \\ ${ }^{1}$ National Institute of Standards and Technology, Gaithersburg, Maryland 20899 \\ ${ }^{2}$ Department of Materials Science and Engineering, University of Maryland, College Park, Maryland 20742 \\ ${ }^{3}$ Department of Chemistry, University of Tennessee, Knoxville, Tennessee 37996
}

Received 23 November 2004; revised 2 August 2005; accepted 13 November 2005

DOI: 10.1002 / polb.20726

Published online in Wiley InterScience (www.interscience.wiley.com).

\begin{abstract}
Poly(ethylene oxide) (PEO), soluble in both aqueous and organic solvents, is one of the most intriguing polymers. PEO solution properties have been extensively studied for decades; however, many of the studies have focused on specific properties, such as clustering, of PEO in aqueous solutions, and the behavior of PEO in organic solvents has not been adequately explored. The results presented here demonstrate that PEO crystallizes into a lamellar structure in ethyl alcohol after the mixture is quenched to room temperature from a temperature above the crystal melting point. Above the melting temperature, PEO completely dissolves in ethyl alcohol, and the mixture exhibits regular polymer solution thermodynamic behavior with an upper critical solution temperature (UCST) phase diagram. Remarkably, the UCST phase boundary is significantly below the melting temperature, and this indicates that the system undergoes a crystallization process before the phase separation can occur upon cooling and, therefore, possesses an unusual phase transition. The phase transition from the crystalline state to the miscible solution state is reversible upon heating or cooling and can be induced by the addition of a small amount of water. ( $\odot 2005$ Wiley Periodicals, Inc. J Polym Sci Part B: Polym Phys 44: 557-564, 2006
\end{abstract}

Keywords: crystalline; ethyl alcohol; phase transition; poly(ethylene oxide)

\section{INTRODUCTION}

Poly(ethylene oxide) (PEO), a semicrystalline polymer and a unique member of the polyepoxides $\left\{\left[\left(\mathrm{CH}_{2}\right)_{m} \mathrm{O}\right]_{n}\right.$ with $\left.m=2\right\}$, is soluble in both aqueous and organic solvents ${ }^{1,2}$ and is, thus, one of the most intriguing polymers whose solution properties have been extensively studied ${ }^{3-20}$ both experimentally ${ }^{3-11,17-20}$ and theoretically ${ }^{12-16}$ for decades. Recently, there has been a large amount of research interest in the $\mathrm{PEO} /$ water system. This interest is due to the similarity of the basic

Correspondence to: D. L. Ho (E-mail: derek.ho@nist.gov) Journal of Polymer Science: Part B: Polymer Physics, Vol. 44, 557-564 (2006) @ 2005 Wiley Periodicals, Inc. interactions (hydrogen-bonding and hydrophobic interactions) involved in $\mathrm{PEO} /$ water and protein/ water systems. The $\mathrm{PEO} /$ water system is a simple model system (synthetic analogue) for studying fundamental biomolecular interactions in which hydrogen-bonding and hydrophobic interactions play important roles, such as protein folding and stabilization. Consequently, most of the studies have been focused on PEO aqueous solutions, ${ }^{3-16}$ and relatively few attempts ${ }^{7,17-20}$ have been conducted to examine the properties of $\mathrm{PEO}$ in organic solvents.

It has been reported that PEO dissolves in methanol in forms of single molecules and clusters, ${ }^{18}$ whereas it crystallizes in toluene and $N, N$ dimethylacetamide. ${ }^{20}$ The understanding of the 
behavior of $\mathrm{PEO}$ in organic solvents is still incomplete, and so it would be interesting and important to perform systematic studies on mixtures of PEO and organic solvents. In addition, our previous studies on the clustering of PEO in water ${ }^{9-}$ ${ }^{11}$ have led us to explore the solution properties of PEO in conventional organic solvents, such as methyl alcohol (methanol), ethyl alcohol (ethanol), benzene, chloroform, and xylene. Among the solvents studied, the mixture of PEO and ethanol exhibits rather unusual and unique phase behavior, which is the subject of this article, observed with differential scanning calorimetry (DSC), small-angle neutron scattering (SANS), and confocal optical microscopy techniques.

\section{EXPERIMENTAL}

To enhance the neutron contrast, ${ }^{21}$ protonated linear PEO $\left[\mathrm{HO}-(\mathrm{C}-\mathrm{C}-\mathrm{O})_{n}-\mathrm{CH}_{3}\right]$, possessing a weight-average molecular weight $\left(M_{\mathrm{w}}\right)$ of $90,000 \mathrm{~g} / \mathrm{mol}$ and a number-average molecular weight $\left(M_{\mathrm{n}}\right)$ of $83,000 \mathrm{~g} / \mathrm{mol}$, with a mass density of $1.13 \mathrm{~g} / \mathrm{cm}^{3}$ (Polymer Source, Inc.), ${ }^{22}$ was dissolved in fully deuterated anhydrous ethanol $\left(\mathrm{C}_{2} \mathrm{D}_{5} \mathrm{OD}\right.$ or ETOD; Cambridge Isotope Laboratories) ${ }^{22}$ at about $70{ }^{\circ} \mathrm{C}$ overnight, and this was followed by the quenching of the mixture to room temperature (ca. $23{ }^{\circ} \mathrm{C}$ ) before the SANS measurements. The overlap concentration ${ }^{23}$ was $C^{*}$ $=M_{\mathrm{w}} /\left(4 \pi R_{\mathrm{G}}^{3} N_{\mathrm{Av}} / 3\right) \approx 0.013 \mathrm{~g} / \mathrm{mL}$, where $R_{\mathrm{G}}$ $\approx 141 \AA$ is the radius of gyration of the $\mathrm{PEO}$ chains ${ }^{8}$ and $N_{\mathrm{Av}}$ is Avogadro's number.

SANS measurements over the wave vector $(\mathbf{q})$ range of $0.0034-0.4415 \AA^{-1}$, with $\lambda=6 \AA$ and $\Delta \lambda /$ $\lambda=0.15$, were carried out with the NG3 $30-\mathrm{m}$ SANS instrument at the National Institute of Standards and Technology Center for Neutron Research. ${ }^{24}$ The scattered intensity was corrected for background and parasitic scattering, placed on an absolute scale with a calibrated secondary standard, and then circularly averaged to yield the scattered intensity, $I(\mathbf{q})$, as a function of $\mathbf{q}$, where $\mathbf{q}=(4 \pi / \lambda) \sin (\theta / 2)(\theta$ is the scattering angle). ${ }^{25}$ The incoherent background from the pure solvent was measured, corrected by the volume fraction displaced by the dissolved $\mathrm{PEO}$, and subtracted from the reduced SANS data. The data points in the $\mathbf{q}$ range from about 0.35 to $0.42 \AA^{-1}$ were then averaged to yield the estimated incoherent background from PEO in the sample, which was subtracted from the data as well.

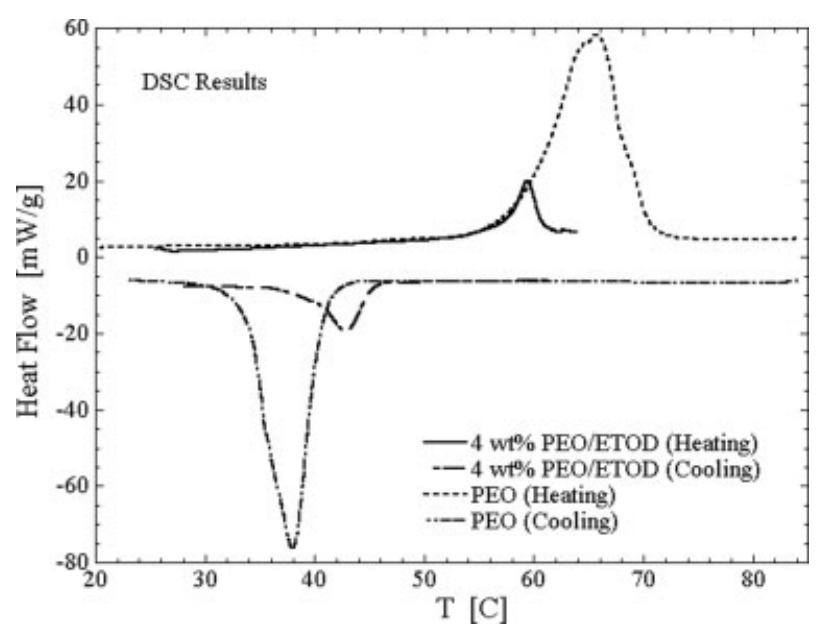

Figure 1. DSC results from neat PEO and a mixture of $4 \mathrm{wt} \%$ PEO in ETOD upon heating and cooling, demonstrating that the $\mathrm{PEO} /$ ethanol mixture also undergoes crystallization and possesses a phase transition.

\section{RESULTS AND DISCUSSION}

PEO does not dissolve in ethanol at room temperature (ca. $23{ }^{\circ} \mathrm{C}$ ) initially. However, mixing at $70{ }^{\circ} \mathrm{C}$ for about $30 \mathrm{~min}$, the mixture became a transparent solution, and this indicated that PEO completely dissolved in ethanol. Upon the quenching of the solution to room temperature for about $15 \mathrm{~min}$, surprisingly, PEO did not simply precipitate from the solvent but formed a uniform, white, pastelike solid, which suggested that PEO formed a new phase in ethanol instead of recovering its initial format. Visually, the pastelike sample turned into a transparent solution again upon reheating to about $40{ }^{\circ} \mathrm{C}$. DSC results shown in Figure 1 attest that the mixture of PEO in ethanol is crystalline under ambient conditions. Both the heating and cooling rates were $2{ }^{\circ} \mathrm{C} / \mathrm{min}$. The melting temperature $\left(T_{\mathrm{m}}\right)$ of the mixture is about $58{ }^{\circ} \mathrm{C}$ upon heating, whereas the mixture starts crystallizing at about $43{ }^{\circ} \mathrm{C}$ when cooling (Fig. 1). In contrast, $T_{\mathrm{m}}$ is about $66{ }^{\circ} \mathrm{C}$ for neat $\mathrm{PEO}$, in good agreement with the literature value. ${ }^{1}$ Confocal optical microscopy images of the same sample given in Figure 2, for instance, show that the crystalline structure that forms in the sample after quenching to room temperature from $70{ }^{\circ} \mathrm{C}$ is network-like over a micrometer length scale. As viewed under the microscope, the network-like structure vanishes rapidly at about $40{ }^{\circ} \mathrm{C}$ and reforms during cooling. These observations show that a phase transition does exist in 


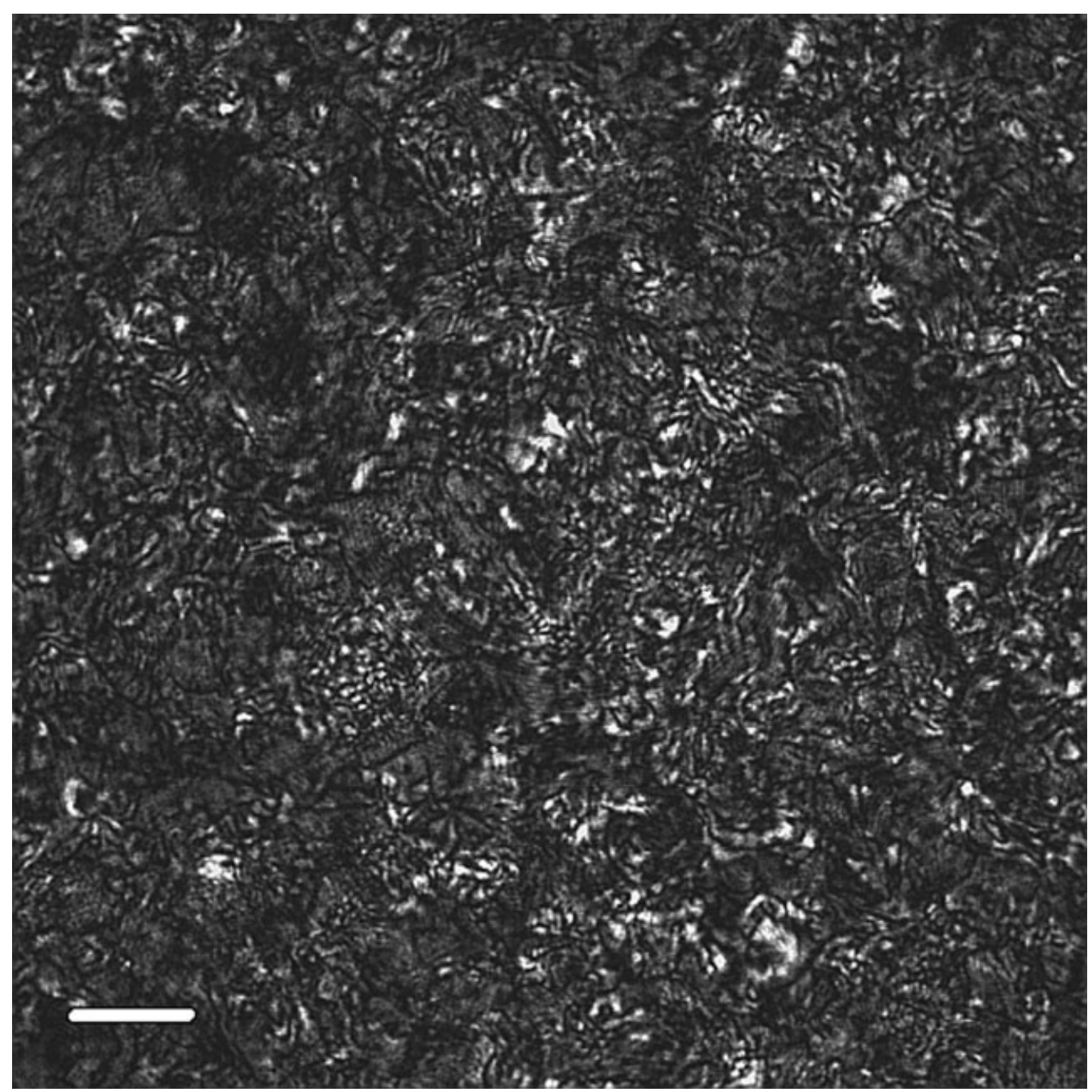

Figure 2. Confocal optical microscopy image of a 4 wt $\%$ PEO/ETOD sample at $23{ }^{\circ} \mathrm{C}$. The picture, taken about $28 \mu \mathrm{m}$ beneath the sample surface, shows that network-like crystalline structures are formed in the sample after the quenching of welldissolved PEO in an ethanol solution from a relatively high temperature (ca. $70{ }^{\circ} \mathrm{C}$ ) to $23{ }^{\circ} \mathrm{C}$. The bright spots in the picture are voids (air bubbles) in the sample. The bar corresponds to a length scale of $20 \mu \mathrm{m}$.

the system and that the behavior is reversible upon heating and cooling.

It is also interesting to note that a $\mathrm{PEO} /$ ethanol mixture at a concentration of 2 wt \% $\mathrm{PEO}$ or higher, which is above $C^{*}$, will not deform even after being shaken strongly at room temperature; this is an indication of the significant strength of the crystallized mixture. Also, no residual ethanol was observed in a mixture of PEO and ethanol at a PEO concentration $\geq 2 \mathrm{wt} \%$, and this suggests that the crystallized particles/units occupy the entire volume of the solution.

To quantitatively characterize the phase behavior as well as the corresponding structure,
SANS experiments were conducted on mixtures of PEO and ETOD at PEO concentrations ranging from 0.5 to $20 \mathrm{wt} \%$ and at temperatures ranging from 23 to $75{ }^{\circ} \mathrm{C}$. The samples were allowed to reach equilibrium for about $40 \mathrm{~min}$ at each temperature before being measured by SANS.

SANS profiles from the PEO/ETOD mixtures at a variety of concentrations at 23 and $45{ }^{\circ} \mathrm{C}$, provided in Figure 3(a,b), respectively, clearly demonstrate the existence of the phase transition. At $23{ }^{\circ} \mathrm{C}$, according to the peaks seen at $\mathbf{q}$ $>0.03 \AA^{-1}$, the profiles confirm that the structures seen by optical microscopy are crystalline in all the samples. The mixtures at $45{ }^{\circ} \mathrm{C}$ exhibit a 
(a)

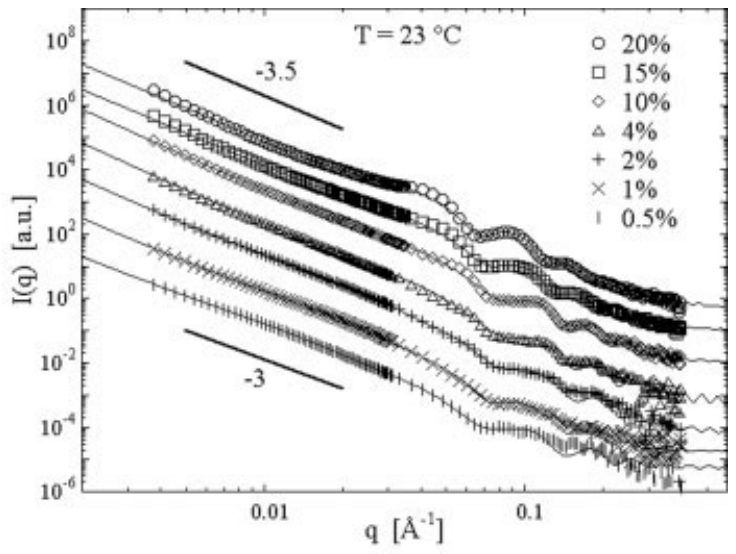

(b)

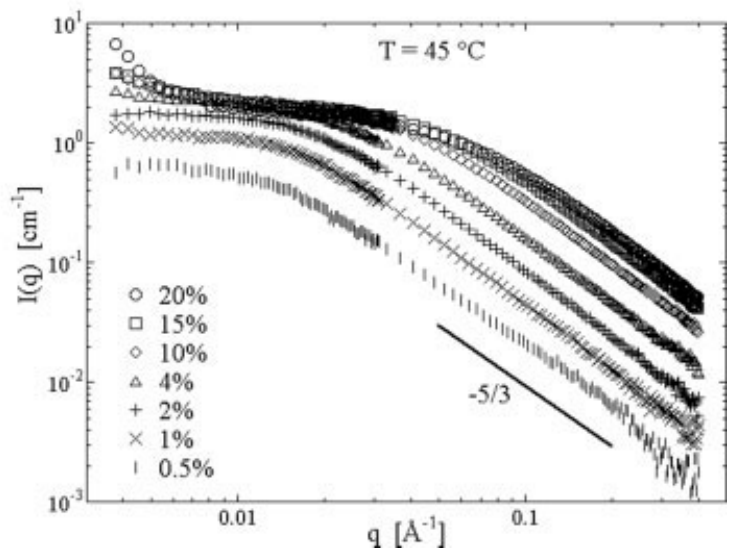

Figure 3. SANS profiles from PEO/ETOD samples with a variety of PEO concentrations. (a) At $23{ }^{\circ} \mathrm{C}$, all the samples possess crystalline structures, with a low-q slope varying from about -3.0 to -3.5 with increasing concentration. The solid curve is the lamellar model fit to the data. The profiles are vertically offset for clarity. (b) At $45{ }^{\circ} \mathrm{C}$, the samples exhibit a regular linear polymer solution behavior thermodynamically, with a highq slope of about $-5 / 3$; this indicates that ethanol is a good solvent for PEO. All the concentrations denote the PEO fraction by weight.

regular polymer solution behavior of linear selfavoiding coils with an excluded volume effect in a good solvent ${ }^{21,26}$ on the basis of the observed high-q slope of about $-5 / 3$.

The solid curve in Figure 3(a) represents the corresponding fit to the data with the model of stacked core/layer platelets, ${ }^{27}$ as illustrated in Figure 4 . In this case, the core containing mainly ethanol is constrained between two layers of crystalline PEO in a single platelet. The platelets further stack together and form a lamellar structure. The lateral dimension of the platelet is on the order of micrometers on the basis of the ultrasmall-angle neutron scattering (USANS) ${ }^{28}$ mea- surements of the mixtures, whereas the thickness of the core and layer and the $d$-spacing between two platelets are on the nanometer scale. A simple term of $A / \mathbf{q}^{m}$, where $A$ is a fitting parameter, is linearly added to the model to describe the lowq slope in Figure 3(a), varying from about -3.0 to -3.5 with increasing concentration, corresponding to the network-like structure on a larger length scale (Fig. 2), which perhaps is further formed by the (relatively local) lamellar crystalline structures (Fig. 4), and indicating that the surface roughness ${ }^{21}$ of the entire crystalline structure decreases with increasing concentration. The neutron scattering length density (NSLD) was calculated to be $6.07 \times 10^{-6}$ and $0.64 \times 10^{-6} \AA^{-2}$ for ETOD and PEO, respectively. During the fitting, the NSLD of the ETOD core was fixed, whereas it was floating for the PEO layers.

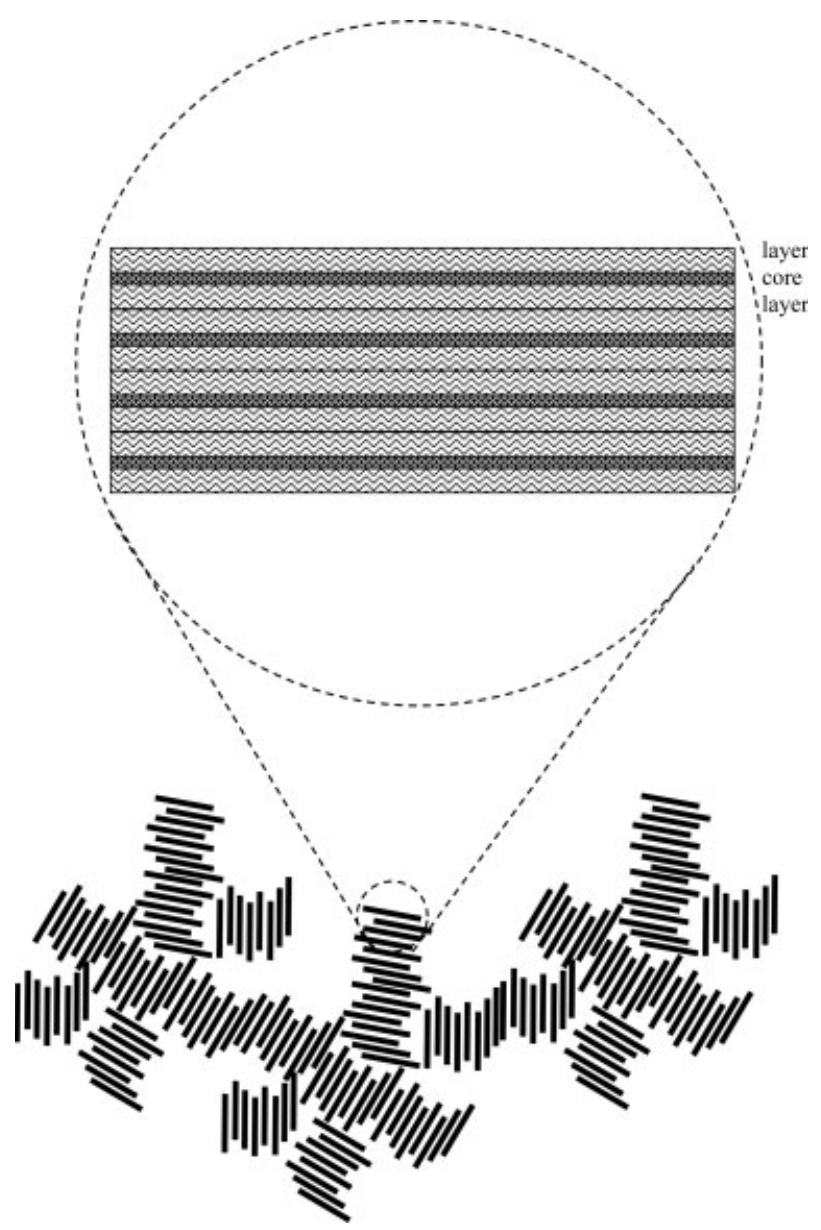

Figure 4. Schematic of the stacked core/layer platelets. The core contains ethanol, whereas the layer is crystalline PEO, forming a lamellar structure locally and a network-like structure on a larger length scale. 
The results obtained from the model fittings [Fig. 5(a)] show that the PEO layer thickness significantly increases, whereas the ethanol core thickness slightly increases, with increasing PEO concentration because of more $\mathrm{PEO}$ and fewer ethanol molecules being present in the mixture with increasing PEO concentration. Therefore, the $d$-spacing of the lamellar structure (total thickness of a platelet) linearly increases with increasing PEO concentration, as demonstrated
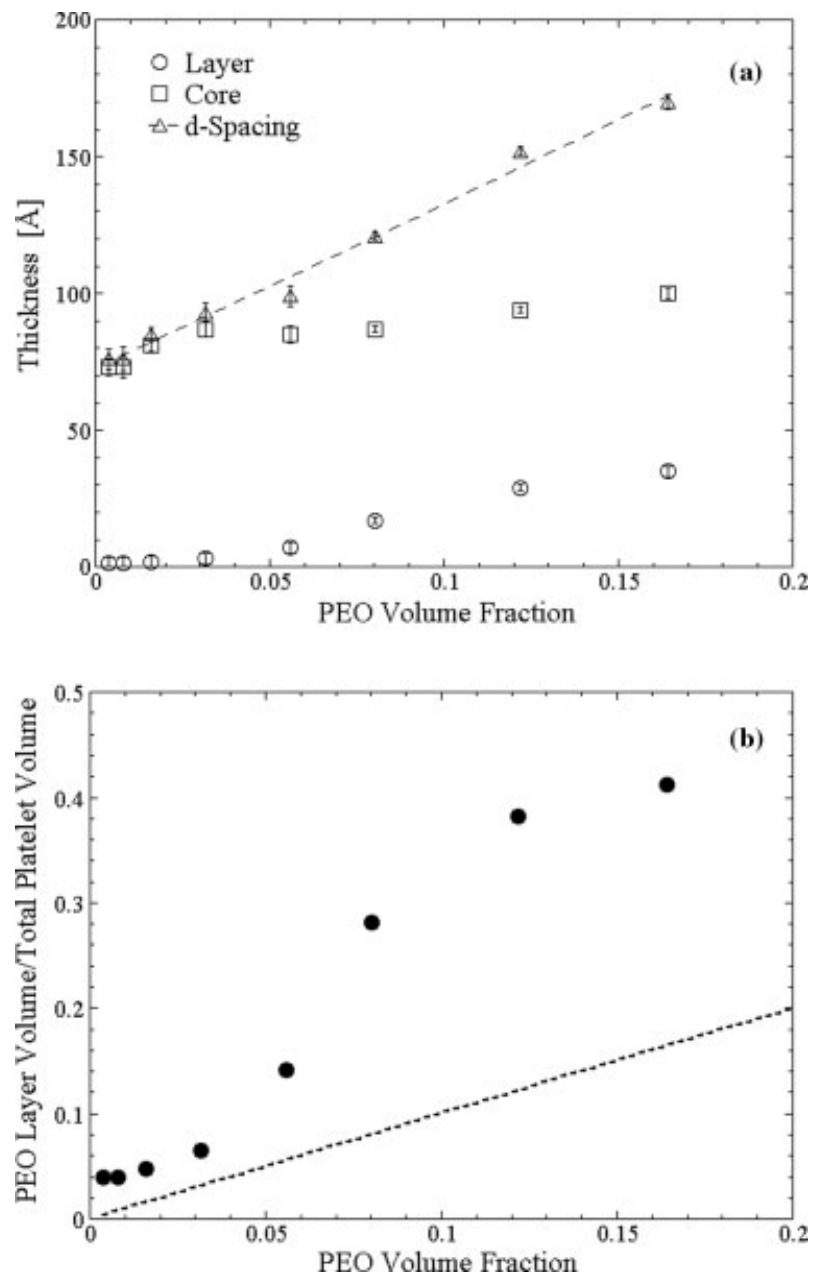

Figure 5. (a) Results regarding the thickness of the core, layer, and $d$-spacing revealed from the model fits given in Figure 3(a). The dashed line is a linear fit to the $d$-spacing data points. (b) Ratio of the total layer volume to the total volume within an individual core/ layer platelet calculated from the fitting versus the corresponding PEO concentration (volume fraction). The ratio was expected to be similar or identical to its corresponding PEO volume fraction (the dashed line). A significant deviation from the expected value implies that the crystalline-layer section contains both PEO chains and ethanol molecules. in Figure 5(a). Another intriguing point is that the ratio of the total layer volume to the total volume within an individual platelet, expected to be identical or similar to the corresponding PEO volume fraction in the mixture, dramatically deviates from its expected value (the dashed line) with increasing PEO concentration, as shown in Figure 5(b). In addition, the NSLD of the PEO layer section varies from about $1 \times 10^{-6}$ to 3 $\times 10^{-6} \AA^{-2}$ with increasing PEO concentration. This implies that the crystalline-layer section contains not only crystalline PEO but also a significant number of ethanol molecules.

The chemical structures of PEO monomers [- $\left.\left(\mathrm{CH}_{2} \mathrm{CH}_{2} \mathrm{O}\right)-\right]$ and ethanol molecules $(\mathrm{H}-$ $\left.\mathrm{CH}_{2} \mathrm{CH}_{2} \mathrm{O}-\mathrm{H}\right)$ are similar. This similarity in the molecular structure at the monomer level makes ethanol a good solvent for PEO at a temperature above $T_{\mathrm{m}}$ in the miscible solution state, in which the PEO chains are essentially surrounded by ethanol molecules possessing a very similar chemical structure and a favorable interaction. Initially, PEO does not dissolve in ethanol at room temperature. Upon quenching, the PEO molecules would prefer to separate from the solvent but cannot because those PEO monomers and ethanol molecules cannot distinguish from each other; as a result, the PEO chains are frustrated and have no choice but to form crystalline structures when there are still some ethanol molecules present in the adjacent regions. Perhaps this is why the layer section consists of both ethanol and crystalline PEO; that is, some ethanol is confined by crystalline $\mathrm{PEO}$ within the layer.

Upon heating, the $\mathrm{PEO} /$ ethanol mixtures undergo a crystal melting process, as demonstrated in Figures 1 and 6(a). The local lamellar structure, represented by the high-q peaks [Fig. 6(a)], vanishes rapidly, whereas the long-range network structure persists with increasing temperature. $T_{\mathrm{m}}$ of the mixture can be estimated as the temperature at which the high-q slope reaches $-5 / 3$, corresponding to linear self-avoiding coils completely dissolved and fully swollen in a good solvent. $T_{\mathrm{m}}$ can then estimated to be about 37, 39, 40, and $41{ }^{\circ} \mathrm{C}$ for the mixtures of PEO/ ETOD with 1, 4, 10, and 20 wt \% PEO, respectively, and this is in good agreement with the visual and microscopy observations.

Profiles at $T \geq 45{ }^{\circ} \mathrm{C}$ in Figure 6(b), with the coherent scattered intensity, $I(\mathbf{q})$, decreasing with increasing temperature, indicate that $\mathrm{PEO} / \mathrm{ethanol}$ solutions possess an upper critical solution temperature (UCST) behavior ${ }^{29}$ thermodynamically; 
(a)

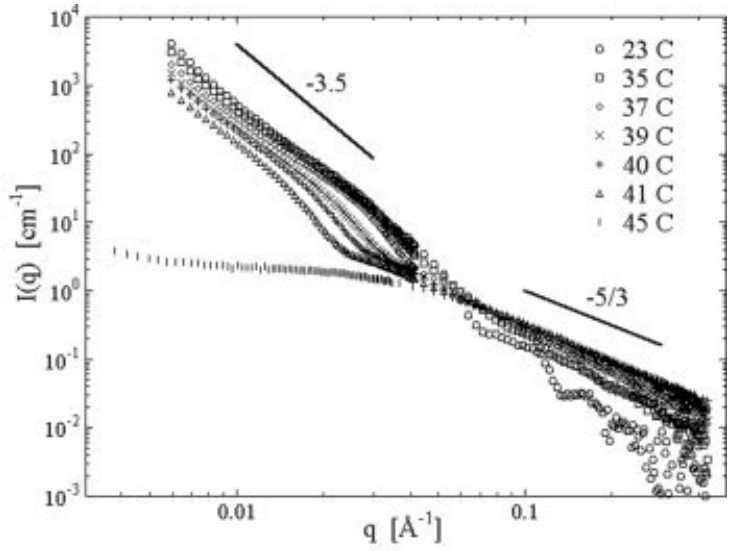

(b)

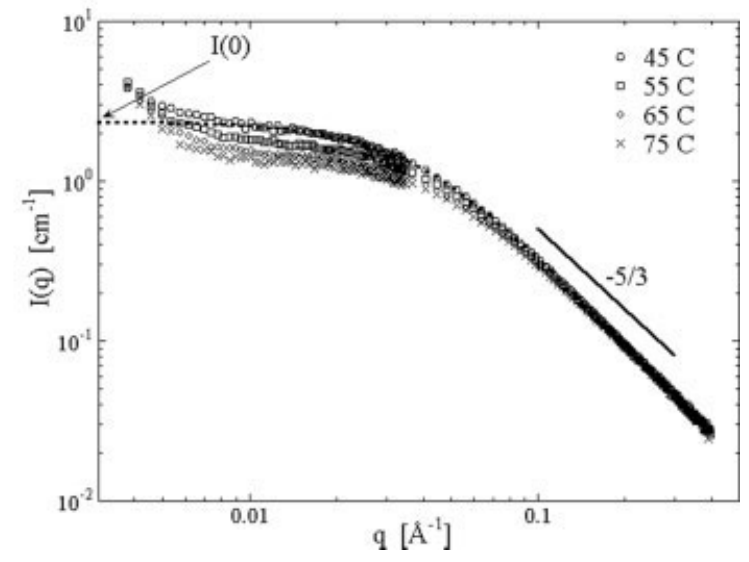

Figure 6. SANS profiles from a $10 \mathrm{wt} \% \mathrm{PEO} / \mathrm{ETOD}$ sample at a variety of temperatures. (a) The sample undergoes a crystal melting process from about 23 to $40{ }^{\circ} \mathrm{C}$. The local structure, corresponding to the feature at relatively high $\mathbf{q}$, vanishes rapidly, whereas the long-range (network) structure, reflected by the low-q profile, persists with a correlation length that increases with increasing temperature. (b) The sample exhibits regular polymer solution behavior at $T$ $\geq 45{ }^{\circ} \mathrm{C}$. The dashed curve through the profile at $T$ $=45{ }^{\circ} \mathrm{C}$, for instance, is the Ornstein-Zernike model fit to the data, yielding the value of $I(0)$ at the intercept.

that is, the solution approaches the two-phase regime upon cooling. Fitting the data points at about $\mathbf{q} \geq 0.01 \AA^{-1}$ to the Ornstein-Zernike equation $^{21,26}$ [dashed curve in Fig. 6(b)], $I(\mathbf{q})=I(0) /(1$ $+\mathbf{q}^{2} \xi^{2}$ ), where $\xi$ denotes the average correlation length between the polymer coils, yields the value of $I(\mathbf{q})$ at $\mathbf{q}=0$, that is, $I(0)$, at each temperature. $I(0)$ is directly related to the second derivative of the free energy of mixing, $\Delta G_{\mathrm{m}}$, in a binary polymer system ${ }^{20,25}$ as $1 / I(0)=\partial^{2} \Delta G_{\mathrm{m}} / \partial \phi^{2}$, where $\phi$ is the polymer concentration. On the phase boundary, $1 / I(0)=\partial^{2} \Delta G_{\mathrm{m}} / \partial \phi^{2}=0$. Thus, in a plot of $1 /$ $I(0)$ versus $1 / T$ ( $T$ in kelvins), extrapolating the data points to $1 / I(0)=0$ yields the phase-separation (spinodal) temperature $\left(T_{\mathrm{s}}\right)$ for each concentration. In Figure 6(b), the upturn seen at low $\mathbf{q}$, which is absent in the profiles from lower concentrations under the same conditions, is mainly due to the clustering behavior of PEO in a concentrated solution. ${ }^{9}$

Figure 7 plots the $T_{\mathrm{m}}$ and $T_{\mathrm{s}}$ values obtained from the SANS measurements at each PEO concentration (volume fraction). The UCST phase boundary is significantly lower than the average $T_{\mathrm{m}}$ value of $40 \pm 1{ }^{\circ} \mathrm{C}$, indicating that the system starts to crystallize before the phase separation can occur upon cooling; that is, the UCST phase boundary is inaccessible thermodynamically. $T_{\mathrm{s}}$ in the UCST phase diagram shown in Figure 7 is relatively low, particularly at low $\mathrm{PEO}$ concentrations, because ethanol is a good solvent for PEO, as discussed previously.

Adding even a small amount of water to the $\mathrm{PEO}$ /ethanol system significantly affects the crystalline structure and the corresponding phase behavior (Fig. 8). The PEO/ETOD/ $\mathrm{D}_{2} \mathrm{O}$ mixtures were prepared through the same procedure introduced earlier in this article. ETOD and $\mathrm{D}_{2} \mathrm{O}$ were mixed at the desired compositions before blending with PEO. The lamellar $d$-spacing increases and the high-q slope approaches $-5 / 3$ with an increasing amount of water (from ETOD/ $\mathrm{D}_{2} \mathrm{O}$ $100 / 0$ to $97 / 3 \mathrm{w} / \mathrm{w}$ ) in the system. At the ETOD/ $\mathrm{D}_{2} \mathrm{O}$ composition of $96 / 4(\mathrm{w} / \mathrm{w})$, the mixture dra-

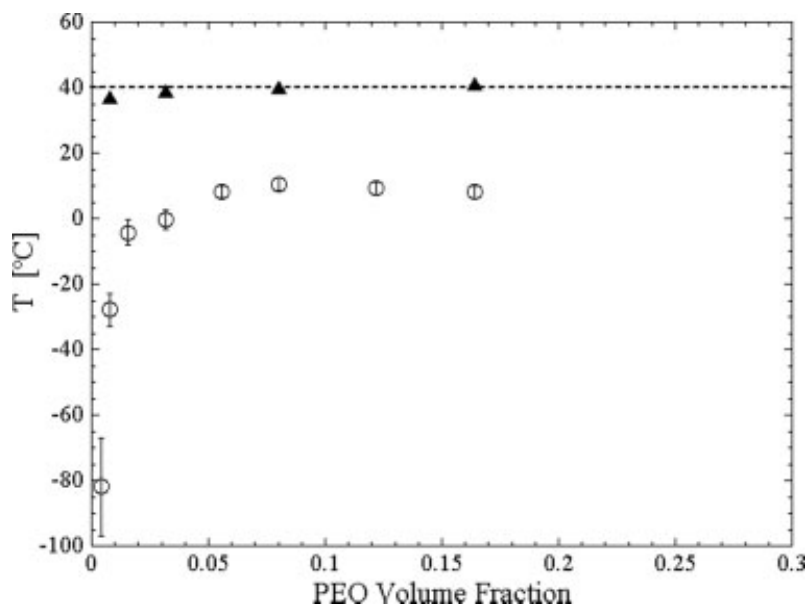

Figure 7. UCST phase diagram of PEO/ETOD. The open circles denote $T_{\mathrm{s}}$ obtained from the SANS data fitting and extrapolation, whereas the solid triangles represent the observed crystal $T_{\mathrm{m}}$ values from the SANS measurements at each concentration studied. The dashed line corresponds to the average $T_{\mathrm{m}}$ value of $40 \pm 1{ }^{\circ} \mathrm{C}$. 


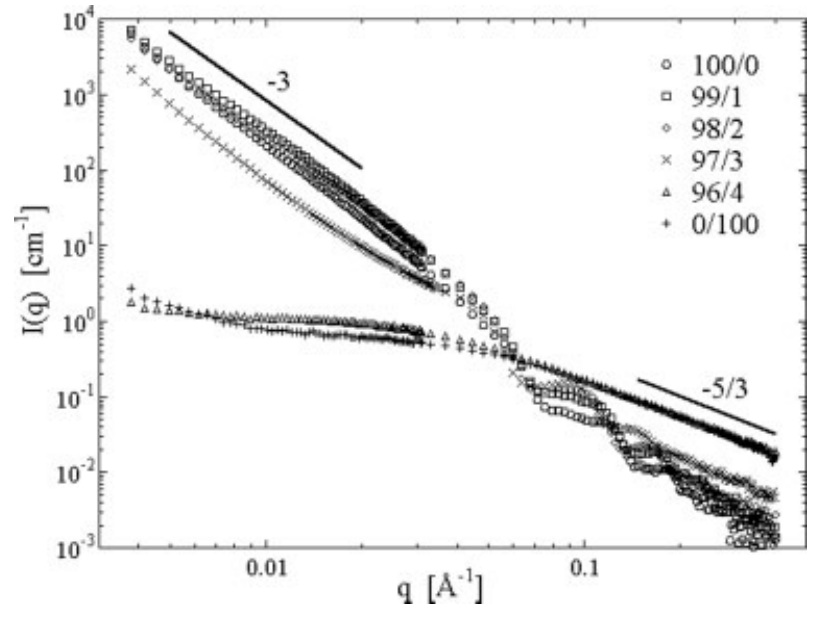

Figure 8. SANS profiles from samples of 4 wt $\%$ $\mathrm{PEO}$ in mixtures of ETOD/ $\mathrm{D}_{2} \mathrm{O}$ at a variety of compositions by weight at $23{ }^{\circ} \mathrm{C}$, demonstrating that adding even a small amount of water to the PEO/ethanol system significantly affects the crystalline structure. At the composition of $96 / 4$ (w/w) ETOD/ $\mathrm{D}_{2} \mathrm{O}$, the mixture dramatically recovers the regular linear polymer solution behavior, and this indicates that the phase transition from the crystalline state to the solution state can be induced by the addition of water. The legend indicates the ratio of $\mathrm{ETOD} / \mathrm{D}_{2} \mathrm{O}$ by weight.

matically recovers the regular linear polymer solution behavior at $23{ }^{\circ} \mathrm{C}$, and this indicates that the phase transition from the crystalline state to the solution state can be induced by the addition of water as well as an increase in the temperature.

It has been shown that $\mathrm{PEO}$ chains interact with water molecules via hydrogen bonding. ${ }^{6,9,14-}$ ${ }^{16}$ A recent topological analysis of the water and PEO system ${ }^{16}$ predicts a coil of water molecules around the PEO chain where a water molecule forms a hydrogen bond with each of the two lone pairs of electrons on each of the PEO ether oxygens. Ethanol forms much weaker hydrogen bonds than water. Therefore, it is reasonable to consider that the water molecules added to a PEO/ethanol mixture preferentially form hydrogen bonds with the PEO chains, resulting in the change observed in the crystalline structure formed by PEO as a function of the water amount added, as illustrated in Figure 8. Once the backbone of a PEO chain has a full complement of hydrogen-bonded water molecules, it loses its ability to crystallize, and consequently, the $\mathrm{PEO} / \mathrm{ETOD} / \mathrm{D}_{2} \mathrm{O}$ mixture behaves as a regular polymer solution. For $4 \mathrm{wt} \% \mathrm{PEO}$, solvents of $97 / 3$ and $96 / 4(\mathrm{w} / \mathrm{w})$ ETOD/ $\mathrm{D}_{2} \mathrm{O}$ correspond to about 1.6 and $2.1 \mathrm{D}_{2} \mathrm{O}$ molecules per PEO mono- mer, respectively. These ETOD/ $\mathrm{D}_{2} \mathrm{O}$ compositions bracket the expected value of 2 water molecules per PEO monomer, corresponding to a critical ETOD $/ \mathrm{D}_{2} \mathrm{O}$ composition of about $96.2 / 3.8(\mathrm{w} / \mathrm{w})$.

\section{CONCLUSIONS}

The findings presented in this article demonstrate that PEO crystallizes and forms a lamellar structure locally as well as a network-like structure on a large length scale in ethanol when a well-mixed solution is quenched at a temperature higher than the crystal $T_{\mathrm{m}}$ value to room temperature. The thermodynamic phase diagram extrapolated from the $\mathrm{PEO} /$ ethanol solutions at temperatures equal to or higher than $45^{\circ} \mathrm{C}$ shows a UCST, which is about $30{ }^{\circ} \mathrm{C}$ below the average $T_{\mathrm{m}}$ of about $40{ }^{\circ} \mathrm{C}$ and, therefore, is not accessible experimentally. The phase transition from the crystalline state to the miscible solution state is reversible upon heating or cooling and can be induced by the addition of water. Extensive studies on the effects of water on the PEO/ethanol system are in progress, and the results will be reported in a future publication.

This work is based on activities supported by the National Science Foundation under agreement no. DMR-9986442. The authors thank L.-P. Sung at the National Institute of Standards and Technology for assistance with the confocal optical microscopy.

\section{REFERENCES AND NOTES}

1. Polymer Handbook, 2nd ed.; Brandrup, J.; Immergut, E. H., Eds.; Wiley: New York, 1974.

2. Molyneux, P. Water-Soluble Synthetic Polymers: Properties and Behavior; CRC: Boca Raton, FL, 1983.

3. Gadd, G. E. Nature 1968, 217, 1040.

4. Cox, L. R.; Dunlop, E. H.; North, A. M. Nature 1974, 249, 243.

5. Devanand, K.; Selser, J. C. Nature 1990, 343, 739 .

6. Cook, R. L.; King, H. E., Jr.; Peiffer, D. G. Phys Rev Lett 1992, 69, 3072.

7. Kinugasa, S.; Nakahara, H.; Fudagawa, N.; Koga, Y. Macromolecules 1994, 27, 6889.

8. Polverari, M.; van de Ven, T. G. M. J Phys Chem 1996, 100, 13687.

9. Hammouda, B.; Ho, D. L.; Kline, S. R. Macromolecules 2002, 35, 8578 .

10. Ho, D. L.; Hammouda, B.; Kline, S. R. J Polym Sci Part B: Polym Phys 2003, 41, 135. 
11. Hammouda, B.; Ho, D. L.; Kline, S. R. Macromolecules 2004, 37, 6932 .

12. Karlström, G. J Phys Chem 1985, 89, 4962.

13. Engkvist, O.; Karlström, G. J Phys Chem 1997, 101, 1631.

14. Smith, G. D.; Bedrov, D.; Borodin, O. Phys Rev Lett 2000, 85, 5583.

15. Dormidontova, E. E. Macromolecules 2002, 35, 987.

16. Aray, Y.; Marquez, M.; Rodriguez, J.; Vega, D.; Simon-Manso, Y.; Coll, S.; Gonzalez, C.; Weitz, D. A. J Phys Chem B 2004, 108, 2418.

17. Booth, C.; Devoy, C. J Polym 1971, 12, 309.

18. Zhou, P.; Brown, W. Macromolecules 1990, 23, 1131.

19. Kinugasa, S.; Nakahara, H.; Kawahara, J.-I.; Koga, Y.; Takaya, H. J Polym Sci Part B: Polym Phys 1996, 34, 583.

20. Sasaki, T.; Miyazaki, A.; Sugiura, S.; Okada, K. Polym J 2002, 34, 794.

21. Higgins, J. S.; Benoît, H. C. Polymers and Neutron Scattering; Oxford: New York, 1994.

22. Certain commercial materials and equipment are identified in this article to specify adequately the experiment procedure. In no case does such identification imply recommendation by the $\mathrm{Na}$ tional Institute of Standards and Technology nor does it imply that the material or equipment identified is necessarily the best available for this purpose.

23. Boyd, R. H.; Phillips, P. J. The Science of Polymer Molecules; Cambridge: New York, 1993.

24. Glinka, C. J.; Barker, J. G.; Hammouda, B.; Krueger, S.; Moyer, J. J.; Orts, W. J. J Appl Crystallogr 1998, 31, 430.

25. Cold Neutron Research Facility at the National Institute of Standards and Technology. NG3 and NG7 30-Meter SANS Instruments Data Acquisition Manual; National Institute of Standards and Technology: Gaithersburg, MD, 2003.

26. Doi, M. Introduction to Polymer Physics; Oxford: New York, 1996.

27. Ho, D. L.; Briber, R. M.; Glinka, C. J. Chem Mater 2001, 13, 1923.

28. Drews, A. R.; Barker, J. G.; Glinka, C. J.; Agamalian, M. Phys B 1998, 241, 189.

29. Physical Properties of Polymers Handbook; Mark, J. E., Ed.; American Institute of Physics: New York, 1996. 